\title{
Economics
}

The Open-Access, Open-Assessment E-Journal

Vol. 12, 2018-37 | June 18, 2018 | http://dx.doi.org/10.5018/economics-ejournal.ja.2018-37

\section{Exchange rates expectations and chaotic dynamics: a replication study}

Jorge Belaire-Franch

\begin{abstract}
In this paper the author analyzes the behavior of exchange rates expectations for four currencies, by considering a re-calculation and an extension of Resende and Zeidan (Expectations and chaotic dynamics: empirical evidence on exchange rates, Economics Letters, 2008). Considering Lyapunov exponent-based tests results, they are not supportive of chaos in exchange rates expectations, although the so-called $0-1$ test strongly supports the chaos hypothesis.
\end{abstract}

(Replication Study)

JEL C12 C15

Keywords Deterministic chaos; exchange rates; expectations; Lyapunov exponents;

$0-1$ test

\section{Authors}

Jorge Belaire-Franch, University of Valencia, Spain, jorge.belaire@uv.es

Citation Jorge Belaire-Franch (2018). Exchange rates expectations and chaotic dynamics: a replication study. Economics: The Open-Access, Open-Assessment E-Journal, 12 (2018-37): 1-8. http://dx.doi.org/10.5018/economics-ejournal.ja.2018-37 


\section{Introduction}

Resende and Zeidan (2008) test for deterministic chaos in exchange rates expectations for four currencies, by means of Fernández-Rodríguez et al. (2005) test, FSA hereafer. Their work, however, presents some formal drawbacks. In the next sections, We re-analyze the problem at hand more accurately and we apply alternative statistical techniques.

Methods are described on Sections 2 through 4 . Section 5 briefly reviews the original paper and section 6 presents the results with a discussion. Section 7 concludes.

\section{FSA test}

The null hypothesis of the test is deterministic chaos. Given a time series of length $T,\left\{y_{t}\right\}_{t=1}^{T}, F S A$ suggest the estimation of the dominant Lyapunov exponent, $\lambda$, to be the bootstrap aggregation, or bagging, of 100 block bootstrap samples of the largest Lyapunov exponents.

Since the largest Lyapunov exponent does not increase when increasing the sample size in a deterministic process, but increases otherwise, FSA propose using $\left\langle\lambda_{\max }\left(T_{i}\right)\right\rangle$ to test for the stability of the largest Lyapunov exponent, where $T_{i}$ is the size of block $i$. This non-increasing property of the largest Lyapunov exponent with the sample size for chaotic processes may be tested by the regression:

$$
\left\langle\lambda_{\max }\left(T_{i}\right)\right\rangle=\alpha_{0}+\alpha_{1} T_{i}+\varepsilon_{T_{i}}
$$

for $T_{i}=T_{1}, \ldots, T_{r}=T$, where $r$ is the number of blocks. Now our interest is on testing the null hypothesis $H_{0}: \alpha \leq 0$. The statistic is $\hat{\alpha}_{1} / s_{1}$ where $s_{1}$ is the standard error of $\hat{\alpha}_{1}$, computed through the Newey-West HAC covariance matrix estimator. ${ }^{1}$

In order to estimate the Lyapunov exponents, we will compute Rosenstein's et al. (1995) algorithm, known as "direct method", with the parameters selected as suggested in FSA (2005).

\section{Shintani and Linton (2004) test}

Let $\left\{y_{t}\right\}_{t=1}^{T}$, be a random scalar sequence generated by the nonlinear autoregressive model:

$$
y_{t}=\theta_{0}\left(y_{t-1}, \ldots, y_{t-d}\right)+u_{t}
$$

where $\theta_{0}: \mathbb{R}^{d} \rightarrow \mathbb{R}$ is a nonlinear dynamic map and $\left\{u_{t}\right\}$ is a sequence of random variables. The model can be expressed in terms of a map with an error vector $U_{t}=\left(u_{t}, 0, \ldots, 0\right)$ and the map function $F: \mathbb{R}^{d} \rightarrow \mathbb{R}^{d}$ such that

$$
Z_{t}=F\left(Z_{t-1}\right)+U_{t}
$$

where $Z_{t}=\left(y_{t}, \ldots, y_{t-d+1}\right)^{\prime} \in \mathbb{R}^{d}$. Let $J_{t}$ be the Jacobian of the map $F$ evaluated at $Z_{t}$. We can obtain the neural network estimator of the largest Lyapunov exponent. This is the so-called

\footnotetext{
1 We are grateful to Professor Andrada-Félix for providing the computer code.
} 
"Jacobian method". Now we distinguish between the sample size $T$ used for estimating the Jacobian $\hat{J}_{t}$ and the block length $M$, which is the number of evaluation points used for estimating the Lyapunov exponent.

Our interest is to test the null hypothesis $H_{0}: \lambda \geq 0$ against the alternative $H_{1}: \lambda<0$, therefore the test is one-sided. Given a consistent estimate tandard deviation of the estimator $\hat{\lambda}$, $\hat{\Phi}$, the test statistic is:

$$
\hat{t}=\frac{\hat{\lambda}_{M}}{\sqrt{\hat{\Phi} / M}}
$$

Under the null hypothesis, $\hat{t}$ is asymptotically distributed as a $N(0,1)$. We compute the heteroskedasticity and autocorrelation consistent (HAC) covariance matrix estimator. ${ }^{2}$

Three alternative selection procedures can be applied: Full, Block and ES (equally spaced subsamples, see Shintani and Linton, 2004, for the details). In all cases, given the number of hidden units $(r)$, the lag length $(d)$ has been selected based on the Bayesian information criterion. The quadratic spectral kernel with optimal bandwidth has been used for the heteroskedasticity and autocorrelation consistent covariance estimation.

\section{The $0-1$ test}

Gottwald and Melbourne (2004) designed the so-called 0 - 1 test for chaos. Given an observation $\phi(j)$ for $j=1,2, \ldots, N$, the $0-1$ test algorithm comprises the following steps:

1. For $c \in(0, \pi)$, compute the translation variables

$$
p_{c}(n)=\sum_{j=1}^{n} \phi(j) \cos j c, q_{c}(n)=\sum_{j=1}^{n} \phi(j) \sin j c,
$$

for $n=1,2, \ldots, N$.

2. Compute the mean square displacement of the transition variables for several values of $c \in(0, \pi)$ and for $n=1,2, \ldots, n_{\text {cut }}$, where $n_{\text {cut }} \ll N$ :

$$
D_{c}(n)=M_{n}(c)-V_{o s c}(c, n)
$$

where

$$
M_{c}(n)=\frac{1}{N} \sum_{j=1}^{N}\left[p_{c}(j+n)-p_{c}(j)\right]^{2}+\left[q_{c}(j+n)-q_{c}(j)\right]^{2}
$$

and

$$
V_{o s c}=\left(\frac{1}{N} \sum_{j=1}^{N} \phi(j)\right)^{2} \frac{1-\cos n c}{1-\cos c}
$$

2 We are grateful to Professor Shitani for providing the computer code. 
3. Form the vectors $\xi=\left(1,2, \ldots n_{\text {cut }}\right)$ and $\Delta=\left(D_{c}(1), D_{c}(2), \ldots, D_{c}\left(n_{\text {cut }}\right)\right)$ and estimate the asymptotic growth rate as the sample correlation coefficient:

$$
K_{c}=\operatorname{corr}(\xi, \Delta)=\frac{\operatorname{cov}(\xi, \Delta)}{\sqrt{\operatorname{var}(\xi) \operatorname{var}(\Delta)}} .
$$

Webel (2012) computed the test on DAX asset returns, assuming that asset returns time series $\left\{r_{t}\right\}_{t=1}^{N}$ are defined by:

$$
r_{t}=s_{t}+\varepsilon_{t}
$$

where $\left\{s_{t}\right\}$ is the one-dimensional signal, an observable of an underlying $k$-dimensional deterministic dynamical system, and $\varepsilon_{t}$ is the random noise. Webel suggests filtering out noise by wavelet denoising techniques, and then applying the 0-1 test to the denoised series. More specifically, Webel (2012) computed Maximal Overlap Discrete Wavelet Transforms (MODWT) for each returns series of 1655 observations, with four Daubechies filters. In this work we follow his guidelines, and compute the denoised original RZ residuals for two filters: Coiflet and Haar and a coarsest resolution chosen to be $\left[\log _{2}(167)\right]=7$ and a soft thresholding rule with resolution dependent universal thresholds. For each filter, 100 random frequencies are drawn from a uniform distribution on $[\pi / 8,7 \pi / 8]$.

Tests statistics are computed for each frequency, hence the cut-off value is set to $n_{\text {cut }}=17$, as suggested by Gottwald and Melbourne (2009). The final statistic for each filter is the median of these 100 single frequency test statistics.

Compared to direct or Jacobian methods, Gottwald and Melbourne (2009) remark the advantages of the 0-1 test: (i) it is binary. (ii) the nature of the vector field as well as its dimensionality does not pose practical limitations, and (iii) it does not suffer from the difficulties associated with phase space reconstruction. In addition, the computation is fast and straightforward, whereas the Jacobian method involves the optimization of target functions to fit neural networks for a range of parameters combinations. Gottwald and Melbourne (2009) show that the procedure is robust against the presence of noise and it can detect weak chaos. However, contrary to FSA and Shintani and Linton procedures, the 0-1 test does not provide a statistical framework, we just can perform a subjective evaluation of the numerical result: is it close enough to 1 to confirm the chaos hypothesis?

\section{Resende and Zeidan (2008) results}

On page 34 Resende and Zeidan's (2008) Table 1 displays the coefficient and the correspondig p-value. We assume that "coefficient" means $\hat{\alpha}_{1}$ for each currency. However, p-value computation deserves some concern. ${ }^{3}$ As FSA argue, the null hypothesis (chaos) includes a wide range of deterministic chaotic processes. Thus, they compute some critical values for just two sample sizes: 380 and 2000 observations, by combining the empirical distribution of the statistics from 250 replications of three of those processes: Logistic, Henon and Lorenz. In Resende and Zeidan (2008),

3 We will assume that the negative p-value for Japanese Yen and $m=3$ is a typo. 
Table 1: FSA test.

\begin{tabular}{llccccc}
\hline & & DIM2 & DIM3 & DIM4 & DIM5 & DIM6 \\
\hline JY & Coefficient (RZ residuals) & 0.0017 & 0.0002 & -0.0000 & 0.0002 & 0.0002 \\
& Coefficient (RZ 2008) & 0.0010 & 0.0008 & 0.0012 & 0.0005 & -0.0001 \\
& p-value (RZ residuals) & 0.0000 & 0.0240 & 0.0070 & 0.0000 & $0.3390^{*}$ \\
& p-value (RZ 2008) & 0.0002 & -0.0003 & 0.0005 & 0.0002 & $0.8865^{*}$ \\
SF & Coefficient (RZ residuals) & 0.0018 & 0.0013 & 0.0012 & 0.0012 & 0.0006 \\
& Coefficient (RZ 2008) & 0.0007 & 0.0008 & 0.0012 & 0.0005 & 0.0009 \\
& p-value (RZ residuals) & 0.0000 & 0.0000 & 0.0000 & 0.0000 & 0.0019 \\
& p-value (RZ 2008) & 0.0069 & 0.0003 & 0.0006 & 0.0012 & 0.0003 \\
DM & Coefficient (RZ residuals) & 0.0017 & 0.0018 & 0.0013 & 0.0013 & 0.0010 \\
& Coefficient (RZ 2008) & 0.0033 & 0.0017 & 0.0015 & 0.0017 & 0.0010 \\
& p-value (RZ residuals) & 0.0000 & 0.0000 & 0.0000 & 0.0000 & 0.0000 \\
& p-value (RZ 2008) & 0.0009 & 0.0000 & 0.0009 & 0.0007 & 0.0007 \\
BP & Coefficient (RZ residuals) & 0.0011 & 0.0011 & 0.0008 & 0.0011 & 0.0010 \\
& Coefficient (RZ 2008) & 0.0000 & 0.0014 & 0.0007 & 0.0009 & 0.0002 \\
& p-value (RZ residuals) & 0.0000 & 0.0000 & 0.0000 & 0.0000 & 0.0003 \\
& p-value (RZ 2008) & 0.0001 & 0.0006 & 0.0010 & $0.0626^{*}$ & 0.0016 \\
\hline
\end{tabular}

DIM stands for embedding dimension. (RZ) are Resende and Zeidan (2008) results. * indicates chaos evidence.

the sample size is 167 observations, hence they should compute the new empirical distribution. Nevertheless, there is no mention to this issue neither to the mentioned procedure to compute the p-values.

\section{Our results}

The analyzed data, as in Resende and Zeidan (2008), correspond to New York Money Market Survey (NYMMS), regarding expected future exchange rates from 30 traders, at one week horizon, for the British pound (BP), the deutschemark (DM), the yen (JY) and the Swiss franc (SF). The data set comprises 168 observations for each currency. Provided the evidence about unit roots, we compute first differences to render the data stationary. Next, Resende and Zeidan (2008) compute the residuals from fitted ARMA models for each first-differenced currency time series, to calculate FSA test on such residuals. ${ }^{4}$ We compute the statistics directly on the residuals series for embedding dimension ranging from 2 to $6 .^{5}$

In order to perform the calculations, we have used the same embedding dimensions and the same samples cited by RZ. Regarding the HAC estimator, the bandwidth parameter was set equal

\footnotetext{
4 The original residuals have been kindly provided by Rodrigo Zeidan.

5 When fitting an ARMA(p,q) model, the sample size of the residuals is reduced depending on the number of AR lags of the fitted model. This reduction is not accounted for in Resende and Zeidan (2008).
} 
to $n^{(1 / 4)}{ }^{6}$ Finally, the reconstruction delay has been set equal to one, and the subsamples have been constructed as suggested in RZ, by setting $T_{1}=88, T_{2}=95, \ldots, T_{17}=167 .^{7}$

In Resende and Zeidan (2008), there is an inconsistency between their Table 1 results and their conclusions. The computed p-values display evidence of chaos for BP at embedding dimension 5, and statistical evidence of chaos for JY at embedding dimension 6. However, they conclude that there is evidence of chaos for DM and JY. Our results do not confirm their results displayed in the text, neither in their Table 1. We just find weak evidence of chaos for JY, for just one embedding dimension as well, but with a rather different p-value: 0.339 vs. $0.886 .{ }^{8}$

Interestingly, in general, our results indicate a marginally stronger rejection of chaotic dynamics than RZ. This could be a result of using a different set of simulated critical values due to a different sample size used in the simulation, or due to a different combination of parameters in the FSA algorithm, or a different number of block bootstrap samples. But we are uncertain due to lack of information about these details.

Regarding Shintani and Linton (2004) test, the lag (d) and the number of hidden units (r) are jointly selected based on BIC. QS kernel with optimal bandwidth is used for the heteroskedasticity and autocorrelation consistent covariance estimation. Based on the Full estimation version of this test, Table 2, there is no evidence of chaos, just marginally for BP, confirming FSA test results. ${ }^{9}$

In addition, we compute the $0-1$ test, Table 3 . The conclusions are sharply different. Hence, with just one exception (JY with $\mathrm{C}(12)$ ), the test statistics are close to one as they range from 0.9891 to 0.9980 .

Table 2: Shintani and Linton test. Full.

\begin{tabular}{cccccc}
\hline \multirow{3}{*}{ BP } & $(d, r)$ & $(1,1)$ & $(2,1)$ & $(3,1)$ & $(4,2)$ \\
& $t$ value & -19.776 & -18.883 & -11.947 & -0.842 \\
& $\mathrm{p}$-value & 0.0000 & 0.000 & 0.000 & $0.200^{*}$ \\
\hline \multirow{3}{*}{$\mathrm{DM}$} & $(d, r)$ & $(1,1)$ & $(2,1)$ & $(3,1)$ & $(4,1)$ \\
& $t$ value & -362.317 & -22.984 & -27.220 & -9.598 \\
& $\mathrm{p}$-value & 0.0000 & 0.000 & 0.000 & 0.0000 \\
\hline \multirow{3}{*}{$\mathrm{JY}$} & $(d, r)$ & $(1,1)$ & $(2,1)$ & $(3,1)$ & $(4,1)$ \\
& $t$ value & -63.579 & -17.383 & -14.719 & -17.407 \\
& $\mathrm{p}$-value & 0.0000 & 0.000 & 0.000 & 0.0000 \\
\hline \multirow{3}{*}{$\mathrm{SF}$} & $(d, r)$ & $(1,1)$ & $(2,1)$ & $(3,1)$ & $(4,1)$ \\
& $t$ value & -35.281 & -15.795 & -15.524 & -9.351 \\
& $\mathrm{p}$-value & 0.0000 & 0.000 & 0.000 & 0.0000 \\
\hline
\end{tabular}

The t-statistics are presented with corresponding one-sided p-values for $H_{0}: \lambda \geq 0$. * indicates chaos evidence.

\footnotetext{
6 This is the default value in the Matlab code kindly provided by Professor Andrada-Félix.

7 In the original work, $T_{17}$ is erroneously set equal to 168 .

8 We contacted the original authors to reconcile the results, but were unable to determine the source of the discrepancies. It should be due to differences in any of the relevant parameters to compute the statistics, since the residuals series used in their work are the same that we have used in this paper.

9 For the estimation based on blocks (Block) and ES, conclusions are identical and are available upon request.
} 
Table 3: $0-1$ test.

\begin{tabular}{lcc}
\hline & Haar & $\mathrm{C}(12)$ \\
\hline BP & 0.9974 & 0.9965 \\
DM & 0.9980 & 0.9891 \\
JY & 0.9877 & 0.7496 \\
SF & 0.9978 & 0.9958
\end{tabular}

The entries are the $0-1$ statistics. Haar is the Haar filter and C(12) is the Coiflet filter with filter length equal to 12 .

They strongly confirm the hypothesis that fluctuations of exchange rates expectations are, at least, partially chaotic.

\section{Conclusions}

In this paper, we have revised and extended Resende and Zeidan (2008) results. We have stressed some important details of the testing process using FSA test. In addition we have applied Shintani and Linton test and the $0-1$ test.

Results of FSA and Shintani and Linton test confirm Resende and Zeidan (2008) conclusions, hence exchange rates expectations are not chaotic. However, results with $0-1$ test point towards chaotic motion (at least partially, after filtering out noise) in all exchange rates expectations.

\section{References}

Fernández-Rodríguez, F., Sosvilla-Rivero, S. and Andrada-Félix, J. (2005). Testing chaotic dynamics via Lyapunov exponents. Journal of Applied Econometrics 20(7), 911-930. https://onlinelibrary.wiley.com/doi/full/10.1002/jae.805

Gottwald, G. A., Melbourne, I. (2004). A new test for chaos in deterministic systems. Proc. R. Soc. A 460, 603-611.http://rspa.royalsocietypublishing.org/content/460/2042/603

Gottwald, G.A., Melbourne, I. (2009). On the implementation of the 0â1 test for chaos. SIAM Journal on Applied Dynamical Systems 8, 129-145.https://epubs.siam.org/doi/abs/10.1137/080718851

Resende, M., Zeidan, R. (2008). Expectations and chaotic dynamics: Empirical evidence on exchange rates. Economics Letters 99(1), 33-35.

https://www.sciencedirect.com/science/article/pii/S016517650700184X

Rosenstein, M.T, Collins, J.J., De Luca, C.J. (1995). A practical method for calculating largest Lyapunov exponents from small data sets. Physica D 65, 117-134. https://www.sciencedirect.com/science/article/pii/016727899390009P 
Shintani, M. and Linton, O. (2004). Nonparametric neural network estimation of Lyapunov exponents and a direct test for chaos. Journal of Econometrics 120(1), 1-33. https://www.sciencedirect.com/science/article/pii/S0304407603002057

Webel, K. (2012).Chaos in German stock returns â New evidence from the 0â1 test. Economics Letters 115(3), 487-489. https://www.sciencedirect.com/science/article/pii/S0165176511006331 


\section{Disclosure Statement}

The author declares that he has no relevant or material financial interests that relate to the research described in this paper. 


\section{Economics}

Please note:

You are most sincerely encouraged to participate in the open assessment of this article. You can do so by either recommending the article or by posting your comments.

\section{Please go to:}

http://dx.doi.org/10.5018/economics-ejournal.ja.2018-37

The Editor 